ORIGINAL ARTICLE

\title{
Alternative mechanisms associated with silencing of CDKN1C in Beckwith-Wiedemann syndrome
}

\author{
N Diaz-Meyer, Y Yang, S N Sait, E R Maher, M J Higgins
}

J Med Genet 2005;42:648-655. doi: 10.1136/jmg.2004.030593

See end of article for authors' affiliations

Correspondence to: Michael J Higgins, Department of Cancer Genetics, Elm and Carlton Streets, Roswell Park Cancer Institute, Buffalo, NY 14263, USA; micháel. higgins@roswellpark.org

Received

29 December 2004

Revised 10 February 2005 Accepted

14 February 2005
Background: Mutations in the imprinted gene CDKN1C account for approximately $10 \%$ of BeckwithWiedemann syndrome (BWS) cases. Fibroblasts from BWS patients with loss of methylation (LOM) at the imprinting control region (ICR) KvDMRI have reduced CDKN1C expression. Another group of BWS patients with downregulated CDKNIC expression but with normal methylation at KvDMRI has been identified.

Objective: To investigate the mechanism of CDKN1C silencing in BWS in these two classes of patients. Methods: The CDKN1C promoter region was analysed for changes in DNA methylation using bisulphite sequencing, and for alterations in chromatin structure using the chromatin immunoprecipitation (ChIP) assay.

Results: There was only spurious CpG methylation of the CDKN1C promoter in fibroblast DNA from both normal individuals and patients with BWS, irrespective of the methylation status of KvDMR1. There was no detectable change in chromatin structure at the CDKN1C promoter in patients with LOM at KvDMR1. BWS patients with downregulated CDKNIC and normal methylation at KvDMRI had depletion of dimethylated $\mathrm{H} 3-\mathrm{K} 4$ and enrichment of dimethylated $\mathrm{H} 3-\mathrm{K} 9$ and $\mathrm{HPl} \gamma$ at the CDKN1C promoter, suggesting that in these cases gene silencing is associated with repressive chromatin changes.

Conclusions: CDKN1C may be downregulated by multiple mechanisms including some that do not involve promoter methylation. In BWS patients with normal methylation at KvDMR1 and reduced expression of CDKN1C, repressive chromatin may play a role, but the absence of methylation and repressive chromatin structure at the CDKN1C promoter in BWS patients with LOM at KvDMR1 argues for a direct role of this epimutation in silencing CDKN1C.
A $\mathrm{n}$ imbalance in the expression of imprinted genes in human chromosome band 11p15.5 (fig 1A), either by mutation or deregulation of genomic imprinting, results in Beckwith-Wiedemann syndrome (BWS), an overgrowth condition where patients have a 1000-fold increased risk of developing embryonal tumours. ${ }^{1} C D K N 1 C$ (also known as $p 57^{K I P 2}$ ) is one of the genes in human chromosome 11 p15.5 that is implicated in BWS. It encodes a cyclin dependent kinase inhibitor involved in the negative regulation of the cell cycle $^{23}$ and is critical during mouse embryogenesis. ${ }^{45}$ CDKNIC is imprinted and primarily expressed from the maternal allele; however, in humans some expression $(5-30 \%)$ is observed from the paternal chromosome. ${ }^{67}$ In BWS, mutations of CDKNIC have been found in $40 \%$ of familial cases and in roughly $5 \%$ of sporadic cases. $^{8-10}$

Chromosome 11 p15.5 contains at least two imprinting control regions (ICRs), which are differentially methylated in the germline and which regulate the imprinted expression of two or more genes. The H19 ICR controls the imprinted expression of the H19 and IGF2 genes (and the Ins gene in mouse) (see the paper by Arney ${ }^{11}$ and references therein), whereas KvDMRl functions by silencing at least eight maternally expressed genes on the paternal chromosome..$^{12-14}$ The exact mode of action of KvDMRl is still unknown. Enhancer blocking assays suggest that KvDMRl may function as a methylation sensitive insulator or silencer. ${ }^{15-18}$ KVDMRl is normally methylated on the maternally inherited allele and unmethylated on the paternal allele. ${ }^{19} 20$ However, a large proportion $(\sim 50 \%)$ of non-UPD BWS patients suffer an epimutation at KvDMRl, namely loss of methylation (LOM) at the maternally inherited allele. ${ }^{19-21}$ These patients have a high occurrence of omphalocele similar to BWS patients with mutations in CDKNIC. ${ }^{10}{ }^{21}$ This observation suggested that LOM at the maternally inherited KvDMRI allele in BWS may result in the downregulation of maternally expressed genes including CDKNIC. Indeed, we have recently demonstrated a strong correlation between LOM at KvDMRl and reduced expression of CDKNIC in BWS patients. $^{22}$ Although we postulate that the silencing of CDKNIC in these patients is a direct consequence of this epimutation at KvDMRl, we cannot exclude the possibility that these two epigenetic phenomena are mechanistically unrelated and simply represent two manifestations of domain-wide epigenetic disruption. In this regard, we have identified some BWS patients with reduced expression of CDKNIC but with apparently normal methylation at KvDMRI.

To gain a better understanding of the mechanisms by which CDKNIC is silenced in BWS, we analysed DNA methylation and chromatin structure in the promoter region of CDKNIC in fibroblasts from normal individuals and patients. We found that the promoter region of CDKNIC was unmethylated in all the normal and BWS patient samples analysed. However, the two subgroups of BWS patients with downregulated CDKNIC were distinguishable on the basis of their chromatin structure at the CDKNIC promoter, indicating different silencing mechanisms.

Abbreviations: BWS, Beckwith-Wiedemann syndrome; ChIP, chromatin immunoprecipitation; ICR, imprinting control region; LOI, loss of imprinting; LOM, loss of methylation; MI, methylation index; RPA, ribonuclease protection assay 


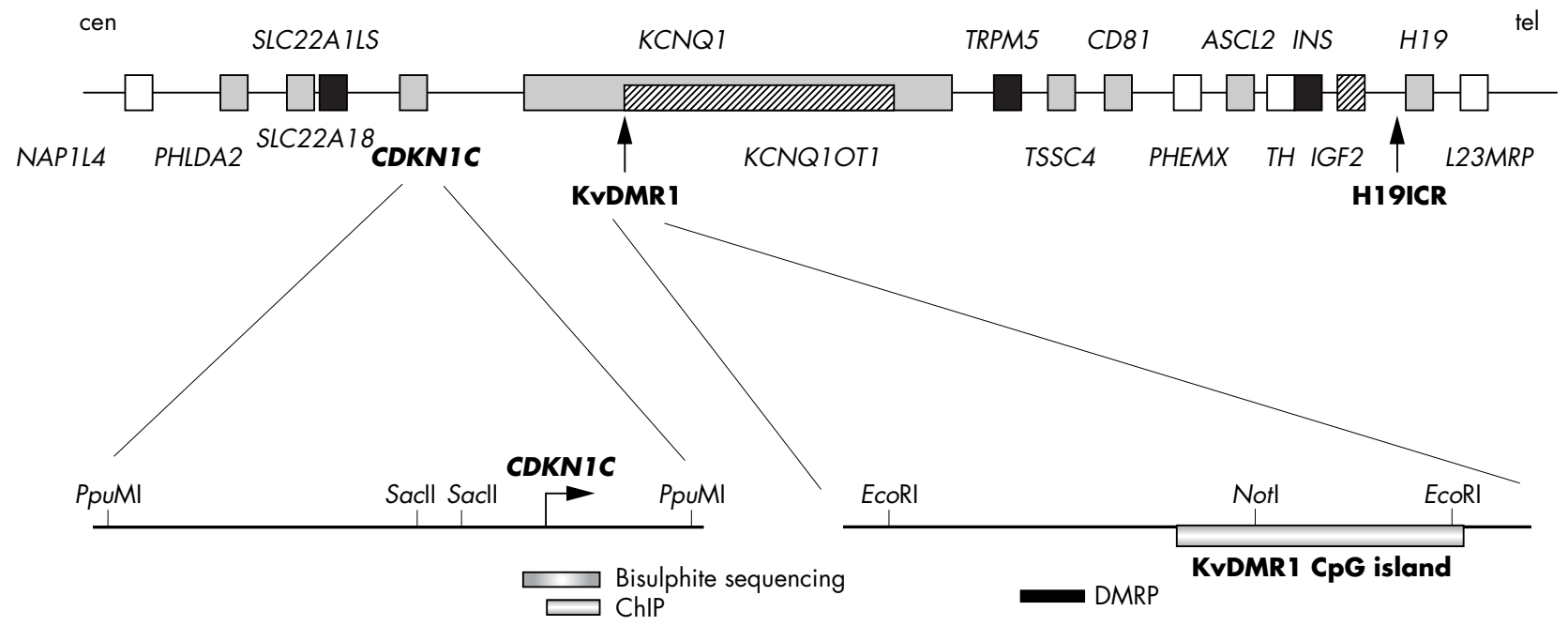

Figure 1 Map of the chromosome 11 p15.5 imprinted domain. Physical map of the $1 \mathrm{Mb}$ imprinted cluster on human chromosome region 11 p15.5. The imprinting status is shown: paternally expressed genes (diagonally stripped box), maternally expressed genes (grey), biallelically expressed genes (white), and unknown (black). Also shown are imprinting control regions (ICR): H19 ICR and KvDMR1. Below the map are enlargements of the CDKN IC (on the left) and KvDMR1 regions (on the right) showing the relative positions of sequences analysed by bisulphite sequencing and chromatin immunoprecipitation (ChIP), and the location of the probe used for Southern blot analysis of KvDMR1. The putative transcription start site for CDKN $1 C^{23}$ is shown with an arrow.

\section{METHODS \\ Methylation analysis of KvDMR 1 and the HI9 ICR region by Southern hybridisation}

Genomic DNA of fibroblast cell lines from normal individuals and BWS patients was digested with EcoRI and the methylation sensitive enzyme NotI. Blots were hybridised with the DMRP probe (fig 1) as described previously. ${ }^{20}$ Hybridisation signals were detected using a Typhoon PhosphorImager and quantitated using ImageJ software (http://rsb.info.nih.gov/ij/). The methylation status (that is, normal or LOM) of a patient was determined by comparing their methylation index (MI; intensity of methylated band/ intensity of unmethylated band) to that of normal controls. For the Southern blot in fig $2 \mathrm{~A}$, the mean (SD) MI of three normal samples was $0.81(0.09)$. Thus methylation at KvDMRl in BWS patients was considered normal if the MI was $0.81 \pm 2 \mathrm{SD}$ or from 0.63 to 0.99 , which is comparable to previous studies. ${ }^{21} 2425$ For analysis of CTCF binding site No 6 of the H19 ICR and the proximal promoter of H19, DNA was digested with Sau96I plus HhaI or with PstI plus SmaI, respectively. The probe for CTCF site No 6 was a 318 base pair (bp) BglII-NcoI fragment corresponding to nucleotides 77958113 of AF125183. The probe for the proximal promoter region of $H 19$ was a cloned polymerase chain reaction (PCR) product encompassing nucleotides 9231-10386 of AF125183.

\section{Bisulphite sequencing}

Genomic DNA was digested with BamHI (Fermentas), which flanks the region analysed by bisulphite sequencing. ${ }^{26}$ Following digestion, DNA was purified by phenol-chloroform extraction and resuspended in $50 \mu \mathrm{H}_{2} \mathrm{O}$. Two micrograms of digested genomic DNA were denatured with $5.5 \mu \mathrm{l}$ of fresh $2 \mathrm{M} \mathrm{NaOH}$ and incubated at $37^{\circ} \mathrm{C}$ for 10 minutes. Bisulphite treatment was carried out at $50^{\circ} \mathrm{C}$ under mineral oil for 16 hours with $30 \mu \mathrm{l}$ of $10 \mathrm{mM}$ hydroquinone (Sigma, St Louis, Missouri, USA) and $520 \mu \mathrm{l}$ of sodium (meta)bisulphite (Sigma) at pH 5.0. Reactions were desalted using the Qiaquick Gel Extraction kit (Qiagen, Valencia, California, USA). Bisulphite treated DNA was eluted in $50 \mu \mathrm{l}$ of water, desulphonated with $5.5 \mu \mathrm{l}$ of $3 \mathrm{M} \mathrm{NaOH}$ for five minutes at room temperature, followed by ethanol precipitation and resuspension in $40 \mu \mathrm{l}$ of water.
Reagents for a $20 \mu \mathrm{l}$ PCR included the following: $1 \mu \mathrm{l}$ of bisulphite treated DNA, I $\mu \mathrm{M}$ PAGE purified primers (IDT DNA) specific for bisulphite treated DNA, $0.2 \mathrm{mM}$ dNTP, $1 \times$ GeneAmp PCR buffer II, $1.5 \mathrm{mM} \mathrm{MgCl}_{2}, 2.5 \%$ DMSO, and 0.5 $\mathrm{U}$ of AmpliTaq Gold DNA polymerase (Applied Biosystems, Foster City, California, USA). Primers for amplification of the CDKNIC promoter region of DNA treated with bisulphite were p57_C.F GGTTGGGYGTTTTATAGGTTA and p57_C.R ACCTAACTÄTCCGATAATAAACTCTTC, as previously described ${ }^{27}$ The following "touchdown" PCR program was used: one cycle of $94^{\circ} \mathrm{C}$ for 10 minutes; 10 "touchdown" cycles of $94^{\circ} \mathrm{C}$ for one minute, $65^{\circ} \mathrm{C}\left(-1.2^{\circ} \mathrm{C}\right.$ per cycle) for one minute, $72^{\circ} \mathrm{C}$ for one minute; 35 cycles of $94^{\circ} \mathrm{C}$ for one minute, $53^{\circ} \mathrm{C}$ for one minute, and $72^{\circ} \mathrm{C}$ for one minute; followed by a final extension cycle of $72^{\circ} \mathrm{C}$ for 10 minutes. Before cloning, amplification was confirmed by running a portion of the PCR products on a 2\% agarose gel. To decrease the chance of stochastically amplified PCR products of bisulphite treated DNA, products from two independent PCRs for each sample were mixed together and cloned into the pCR4-TOPO vector following the manufacturer's recommendations (Invitrogen, San Diego, California, USA). Plasmid DNA was isolated by miniprep using the Qiaprep 96 Turbo BioRobot kit (Qiagen) or the High Pure Plasmid isolation kit (Roche, Indianopolis, Indiana, USA). For every sample analysed by bisulphite sequencing, 10 clones were sequenced with T3 primers using an ABI PRISM 3700 DNA analyser (Applied Biosystems). Sequencing was done by Macrogen (Seoul, Korea). In the alignment process, vector sequences were removed and clone sequences were aligned. A compilation of individual sequenced clones was saved in FASTA format. Methylation status was determined by using the MethTools software (http://genome.imb-jena.de/ methtools).

\section{Ribonuclease protection assay (RPA)}

RPA was carried out as described previously. ${ }^{22}$ Briefly, radioactive RNA probes were synthesised using the MaxiScript T7/T3 kit (Ambion, Austin, Texas, USA) and ${ }^{32} \mathrm{P}-$ UTP (800Ci/mmol) (Perkin-Elmer Life Sciences, Norwalk, Connecticut, USA). The probe used for CDKNIC was from Pharmingen (San Diego, California, USA) and the human 
A

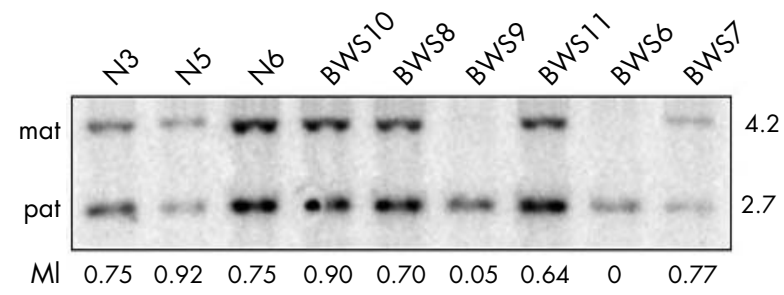

B

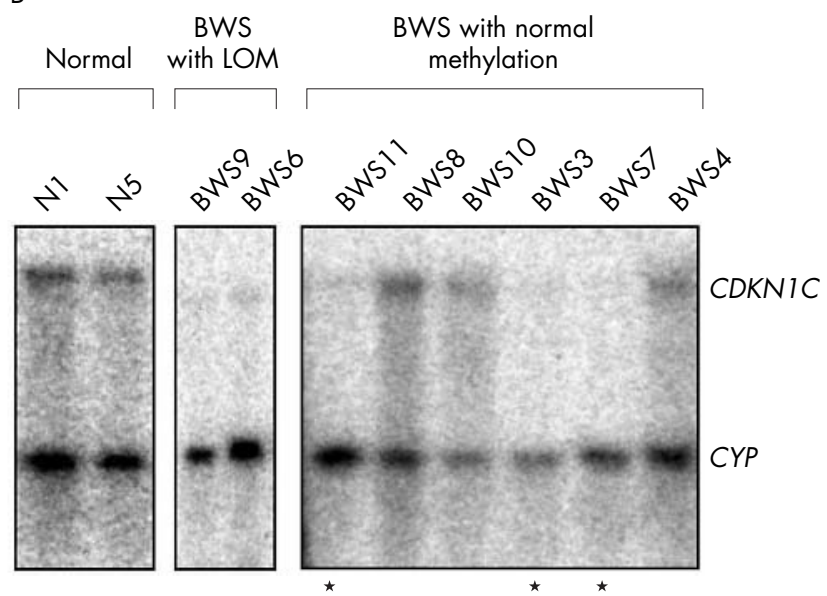

Figure 2 (A) Analysis of methylation status of KvDMR1 by Southern hybridisation. DNA from Beckwith-Wiedemann patient fibroblast cell lines (BWS) and from normal individuals (N) was digested with EcoRI and Notl. Blots were hybridised with the DMRP probe (Smilinich et aPo, fig 1B). The Southern blot analysis differentiates between the methylated $(4.2 \mathrm{~kb})$ and unmethylated $(2.7 \mathrm{~kb})$ alleles. Densitometry analysis of the bands allowed the samples to be classified as normal methylation (methylation index (MI) $0.81 \pm 2 \mathrm{SD}$ or from 0.63 to 0.99 ) or loss of methylation at $\mathrm{KvDMR1}(\mathrm{MI}<0.63$ ). (B) Downregulation of CDKNIC in BWS patients. Ribonuclease protection assay was undertaken as described by Diaz-Meyer et a ${ }^{22}$ with CDKNIC and CYCLOPHILIN (CYP) RNA probes. Asterisks indicate BWS patient samples with normal methylation at KvDMR1 that show downregulation of CDKN1C. For comparison, the results of two BWS patients with LOM at KvDMR1 are included (BWS6 and BWS9). ${ }^{22}$

tri-cyclophillin probe was from Ambion. RPA was carried out with $30 \mu \mathrm{g}$ total RNA using the RPAIII kit, as described by the manufacturer (Ambion) with hybridisation at $55^{\circ} \mathrm{C}$. RNase digestion was done with a 1/100 dilution of the RNaseA/Tl mix. Conditions were such that both probes were in excess. Protected fragments were electrophoresed in 5\% TBE-urea precast Ready Gels (BioRad, Hercules, California, USA).

\section{Chromatin immunoprecipitation (ChIP)}

We used the ChIP assay kit from Upstate Biotechnology (Santa Cruz, California, USA). Between one million and five million cells were fixed with $1 \%$ formaldehyde for 10 minutes at $37^{\circ} \mathrm{C}$ in cell culture media. The formaldehyde cross linked cells in $0.6 \mathrm{ml}$ sodium dodecylsulphate lysis buffer were sonicated for 180 seconds ( 10 seconds on, and five seconds off) on ice using a Branson sonicator with a $2 \mathrm{~mm}$ microtip, and settings of $40 \%$ for output control and $90 \%$ for duty cycle. Sonicated chromatin (200-1000 bp) was centrifuged at $20000 \times g$ for 10 minutes at $4^{\circ} \mathrm{C}$, aliquoted, snap frozen in liquid nitrogen, and stored at $-70^{\circ} \mathrm{C}$. Antibodies obtained from Upstate Biotechnology (Waltham, Massachusetts, USA) included anti-dimethyl histone H3 (lys4) (catalogue No 07030), and anti-dimethyl histone H3 (lys9) (catalogue No 07212). Antibody against HPl- $\gamma$ (catalogue No MAB3450) was from Chemicon International Inc (Temecula, California, USA). Sonicated chromatin was diluted 10-fold in ChIP dilution buffer $(200 \mu \mathrm{l})$, pre-cleared with $80 \mu \mathrm{l}$ salmon sperm $\mathrm{DNA}$ /protein A agarose for one hour at $4^{\circ} \mathrm{C}$ with rotation. A portion of the protein A purified chromatin $(20 \mu \mathrm{l})$ was used to prepare DNA for the "input" sample. Antibodies $(2-5 \mu \mathrm{l})$ were added to the clarified chromatin ( $180 \mu \mathrm{l})$ and incubated overnight with rotation. Sixty microlitres of protein A agarose were added to the antibody-chromatin mix and incubated at $4^{\circ} \mathrm{C}$ for one hour with rotation. The complex was collected by gentle centrifugation and washed three times with ChIP buffers (Upstate Biotechnology), and the bound chromatin was eluted in $500 \mu \mathrm{l}$ of elution buffer. After adding $20 \mu \mathrm{l}$ of $5 \mathrm{M} \mathrm{NaCl}$, protein-DNA cross linking was reversed by heating at $65^{\circ} \mathrm{C}$ for four hours. Samples were treated with proteinase $\mathrm{K}$, purified by MiniElute PCR purification kit (Qiagen), and then eluted in $100 \mu \mathrm{l}$ of low TE buffer ( $1 \mathrm{mM}$
Tris, $0.1 \mathrm{mM}$ EDTA). Chromatin modifications were determined using LDHA and $\beta$ globin as internal controls. All the primes were located in the promoter regions or the first exon. The primers used are the following: CDKNIC.F AGGCCTGA GCGA GCGAGCTAGCCA and CDKNIC.R CTGTCCGGTGGT GGACTCTTCTGCGT; LDHA.F GGGTATGGTTGAGACTCGAGA TGAG and LDHA.R TTGCAGGGTGACTCAGTTCAAATGTA; $\beta$ globin.F GTACTGATGGTATGGGGCCAAGAGA and $\beta$ globin.R GGTCTAAGTGATGACAGCCGTACCTG.

\section{RESULTS}

CDKN $1 C$ expression is reduced in some BWS patients with apparently normal methylation at KvDMR 1

We have previously observed complete concordance between LOM at KVDMRI and the reduction of CDKNIC expression in fibroblasts from BWS patients. ${ }^{22}$ From an expression analysis of six BWS patients with normal methylation at KvDMRI (fig 2A and data not shown), three samples (BWS3, BWS7, BWS11) were identified with decreased expression of CDKNIC using either a ribonuclease protection assay (RPA) (fig 2B) or real time reverse transcriptase PCR (RT-PCR, data not shown). The degree of CDKNIC repression was similar to that observed in BWS patients with LOM at KvDMRl (fig 2B, BWS6 and BWS9).22 These patients all had a normal karyotype and the finding of normal methylation at KvDMRl makes paternal UDP unlikely. Though unexpected, these results suggest an additional mechanism for downregulation of CDKNIC in these BWS patients, independent of KvDMRl methylation.

\section{Silencing of CDKN1C in BWS is not associated with hypermethylation at the promoter region}

A large $\mathrm{CpG}$ island containing the promoter region and most of the gene body of CDKNIC is differentially methylated in mouse but not in humans. ${ }^{6}$ However, it has been shown that CDKNIC becomes biallelically methylated in several human cancers and that this correlates with downregulation of CDKNIC expression. ${ }^{27-30}$ In our earlier study, we showed by Southern blot that methylation is absent at two SacII sites in the promoter region of CDKNIC (fig l) in BWS patients with loss of methylation at KvDMRl and downregulation of 

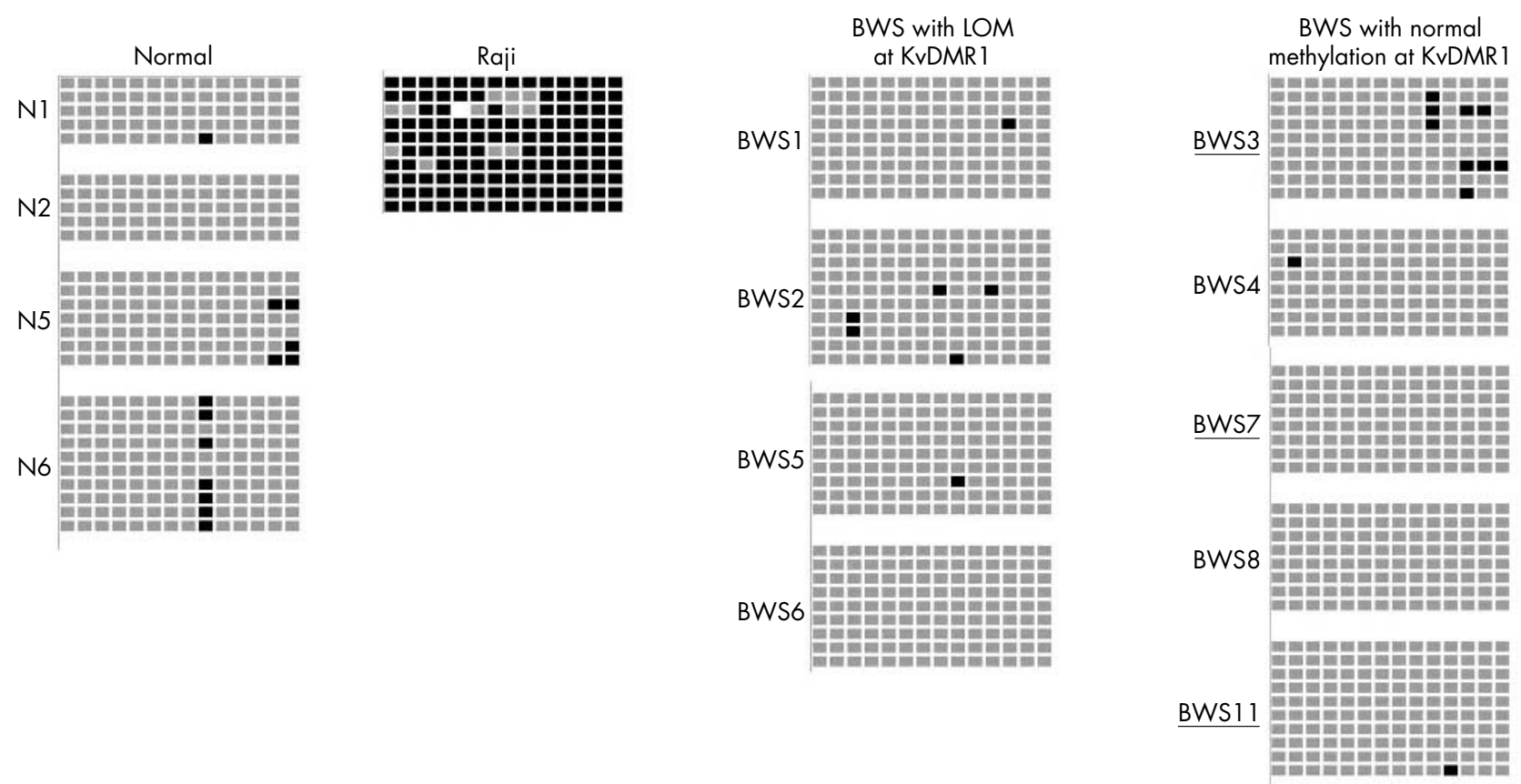

Figure 3 Methylation analysis of the promoter region of CDKNIC by bisulphite sequencing. Bisulphite sequencing was carried out on a region corresponding to the promoter and part of the first exon of the CDKN1C gene and spans a total of 155 bp containing 14 CpGs (fig 1). For a given sample, each line represents the results for a single cloned DNA molecule. Black rectangles represent methylated CpGs, while grey rectangles represent unmethylated CpGs. Except for the tumour cell line Raji, samples were fibroblast DNA from normal individuals and non-UPD Beckwith-Wiedemann syndrome (BWS) patients with and without loss of methylation (LOM) at KvDMR1. BWS cases with reduced expression of CDKN1C but normal methylation at KvDMR1 are underlined.

CDKNIC.22 To rule out the possibility that downregulation of CDKNIC observed in BWS patients simply reflects methylation elsewhere at the CDKNIC promoter, we carried out a comprehensive methylation analysis of the promoter using bisulphite sequencing.

PCR primers were chosen that amplified a $155 \mathrm{bp}$ fragment containing $14 \mathrm{CpG}$ dinucleotides spanning the proximal promoter and transcription start site of $C D K N I C$, and which is consistently methylated in tumour cell lines where the CDKNIC gene is silenced (fragment C in Kikuchi et al ${ }^{27}$ ). PCR products were cloned and 10 clones were sequenced for each sample. As a positive control, the same region of CDKNIC was analysed in Raji, a Burkitt's lymphoma cell line previously shown to be densely methylated at the CDKNIC promoter. $^{27}$ In agreement with the earlier study, all 10 molecules analysed from Raji DNA showed primarily methylated $\mathrm{CpG}$ dinucleotides (fig 3). In contrast all clones from four normal fibroblast lines that gave reliable sequencing information indicated that the CDKNIC proximal promoter region is predominantly unmethylated (fig 3). As no polymorphisms were identified in the CDKNIC promoter region to assess PCR bias, a mixing experiment was undertaken using different amounts of Raji and normal DNA to confirm that we were able to detect methylation at the CDKNIC promoter region in the presence of the unmethylated allele (that is, to rule out PCR bias towards the unmethylated allele). Using COBRA analysis, ${ }^{31}$ methylated alleles were easily detected in PCR products from a 1:9 (Raji:normal) DNA mixture (data not shown), indicating that, if a bias did exist, it favoured amplification of methylated alleles. Despite some sporadic methylation, we found no significant differences in methylation patterns in BWS patients compared with normal controls (fig 3), regardless of KvDMRl methylation status. Importantly, this confirms that there does not appear to be aberrant DNA methylation at the CDKNIC promoter region in BWS patients who show downregulation of $C D K N I C$ associated with LOM at KvDMR 1.22
Considering that we observed primarily unmethylated alleles in the normal and BWS samples, silencing mechanisms other than the hypermethylation observed in some cancers ${ }^{27-30}$ appear to be operating in BWS.

\section{Repressive chromatin in BWS patients with downregulation of CDKN 1 C but normal methylation at KvDMR 1}

As BWS patients with loss of methylation at KvDMRl, and some with normal methylation at KvDMRl, both show downregulation of CDKNIC in a promoter methylation independent fashion, we postulated that chromatin modifications associated with gene silencing might be involved. We first sought to determine whether allele specific differences in chromatin structure were detectable at the CDKNIC promoter by carrying out chromatin immunoprecipitation (ChIP) experiments on somatic cell hybrids containing a single human chromosome 11 of known parental origin. We have previously shown that these hybrids maintain both allele specific gene expression and methylation..$^{32}$ The advantage of these cell lines is that one can examine the chromatin structure of each parental allele independently, even in the absence of a polymorphism. As internal controls, we used the promoter regions of the housekeeping gene $L D H A$ and the erythroid specific $\beta$ globin gene, two chromosome 11 genes assumed to be active and silent, respectively, in the somatic cell hybrids. Figure 4 shows that, compared with the hybrid carrying the paternal chromosome, the CDKNIC promoter in the maternal hybrid was highly enriched in dimethyl H3-K4, a histone modification associated with active genes, ${ }^{33}$ and depleted in HPl $\gamma$, a protein associated with the chromatin of inactive promoters. ${ }^{34}$ In contrast, the hybrid containing the paternally derived chromosome 11 had a threefold lower level of H3-K4 dimethylation, but was enriched for HPl $\gamma$ at the CDKNIC promoter. These results are consistent with the imprinted expression of CDKNIC, which is expressed predominantly from the maternal allele. ${ }^{8}$ Somewhat 
A

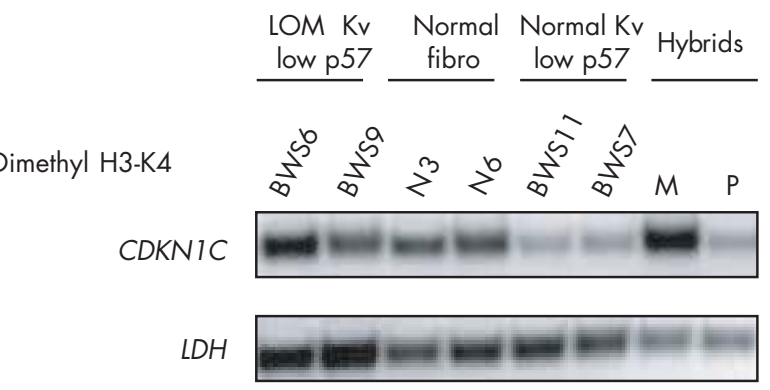

Dimethyl-H3-K9

CDKN1C

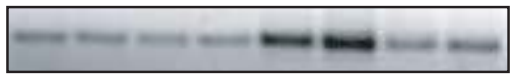

GLOBIN

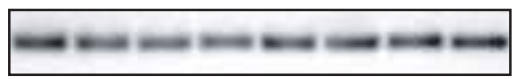

HPI- $\gamma$

CDKNIC

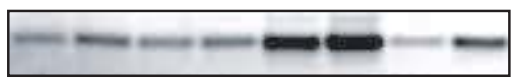

GLOBIN

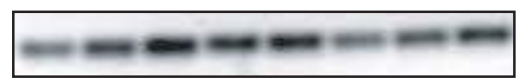

Input

CDKNIC

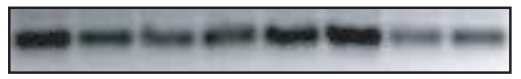

$L D H$

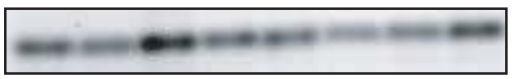

GLOBIN
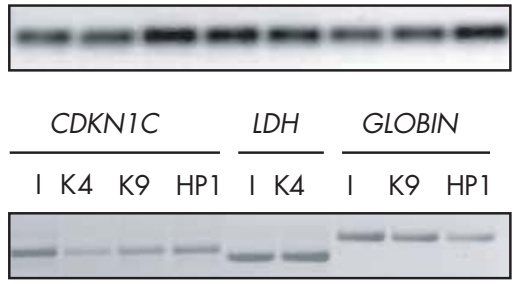

RAll

B

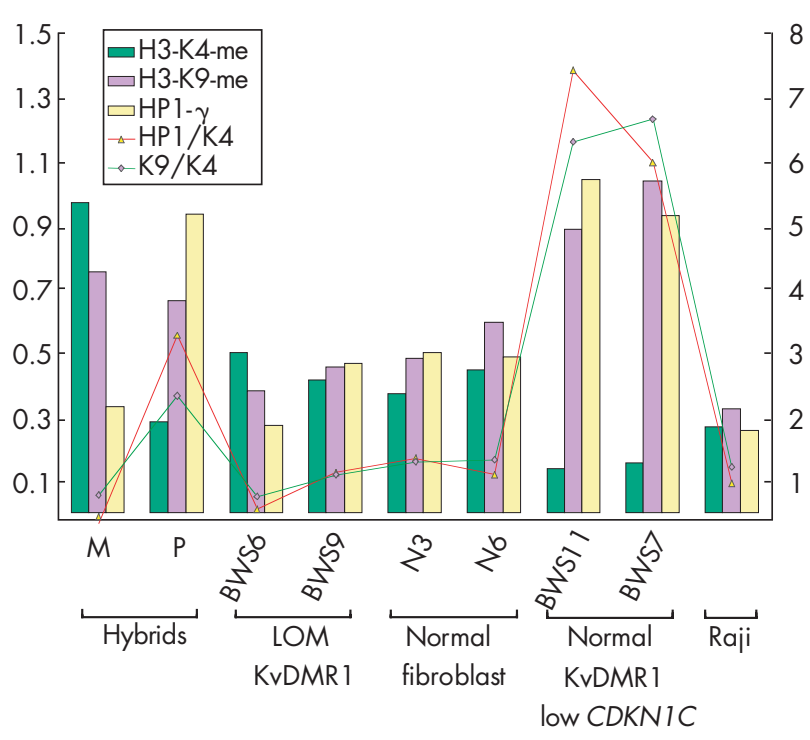

surprisingly, we found little difference between the maternal and paternal $C D K N I C$ promoters with respect to dimethyl H3K9 levels, a histone modification associated with silent chromatin. ${ }^{35}$ (fig $4 \mathrm{~A}$ and $4 \mathrm{~B}$ ).

ChIP analysis using the same antibodies detected no differences at the CDKNIC promoter between fibroblasts from normal individuals and BWS patients with loss of methylation at KvDMRl (fig 4A and 4B). The same analysis was carried out on two of the three BWS samples with reduced $C D K N 1 C$ expression but normal methylation at KvDMRl (the third sample was not available for analysis). Strikingly, cells from these patients showed both decreased levels of H3-K4 dimethylation and enrichment of dimethyl H3-K9 and HPl $\gamma$ (fig 4A and 4B). These data suggest that, compared with normal controls and BWS patients with LOM at KvDMRl, a repressive chromatin structure exists at the CDKNIC promoter in this BWS subgroup which is probably associated with gene silencing. Raji, the Burkitt's lymphoma cell line that is hypermethylated at the CDKNIC promoter (fig 4), ${ }^{27}$ shows a ChIP profile similar to the fibroblast cell lines from normal individuals for all three antibodies analysed. This indicates that even though there is a reduction in CDKNIC expression levels, no change in histone modification was identified. More importantly, this suggests that DNA methylation alone (or in combination with DNA methylation binding proteins) is capable of downregulating CDKNIC without accompanying chromatin changes typical of repressed chromatin. Furthermore, Raji shows normal methylation at the NotI site in KvDMRl as determined by Southern blot (not shown), suggesting that KvDMRl is not associated with the silencing of CDKNIC by hypermethylation.

\section{Downregulation of CDKNIC is not associated with hypermethylation at $\mathrm{H} 19$}

Several mouse models have been constructed that partially recapitulate the complex phenotype of BWS, the most complete being a mouse line having both a null mutation of Cdknlc and loss of imprinting for Igf2 (see Caspary et al ${ }^{36}$ and references therein). Analysis of this double mutant suggested that CdknIc and Igf2 function in the same developmental pathway and are antagonistic. A corollary of this, which is consistent with the high degree of similarity between the mutant mouse phenotype and BWS, is that CDKNIC and IGF2 may synergise to produce some characteristics of this condition. In BWS, loss of imprinting (LOI) at

Figure 4 Analysis of chromatin structure at the promoter region of CDKNIC by chromatin immunoprecipitation (ChIP). (A) ChIP

polymerase chain reaction (PCR) results obtained using antidimethyl H3$\mathrm{K} 4$, antidimethyl $\mathrm{H} 3-\mathrm{K} 9$, and anti HP1- $\gamma$ antibodies. Samples analysed correspond to: (1) fibroblast cell lines from Beckwith-Wiedemann syndrome (BWS) patients (BWS6 and BWS9) with loss of methylation at KvDMRI and with low expression of CDKNIC (LOM Kv/low p57); (2) fibroblast cell lines from normal individuals (N3 and N6); (3) fibroblast cell lines from BWS patients (BWS7 and BWS11) with normal methylation at KvDMR1 and low expression of CDKNIC (normal Kv/ lowp57); (4) human-mouse somatic cell hybrids containing either a maternally derived human chromosome $11(\mathrm{M})$ or a paternally derived human chromosome 11 (P). The Burkitt's lymphoma cell line, Raii, was also analysed. Input (I) corresponds to PCR of DNA isolated from chromatin before immunoprecipitation. (B) Histograms show the relative enrichment (left vertical axis values) of dimethyl H3-K4 (green), dimethyl H3-K9 (purple), and HP1- $\gamma$ (yellow) in the different samples analysed. The relative enrichment for each chromatin modification was determined by comparing the normalised intensity (relative to input) of the bands for CDKNIC dimethyl H3-K4 with that of $L D H A$, and dimethyl H3-K9, or $\mathrm{HPI}-\gamma$, with that of $\beta$ globin. Lines in the graph show the enrichment (right vertical axis values) of silencing associated factors, $\mathrm{HP1}-\gamma$ (red) and dimethyl H3-K9 (green) relative to dimethyl H3-K4. Note the dramatic enrichment of $\mathrm{HPI}^{-} \gamma$ and dimethyl H3-K9 with respect to dimethyl H3-K4 in the paternal chromosome hybrid and in BWS patients with normal methylation at KvDMR1 and downregulation of CDKN1C. 
A
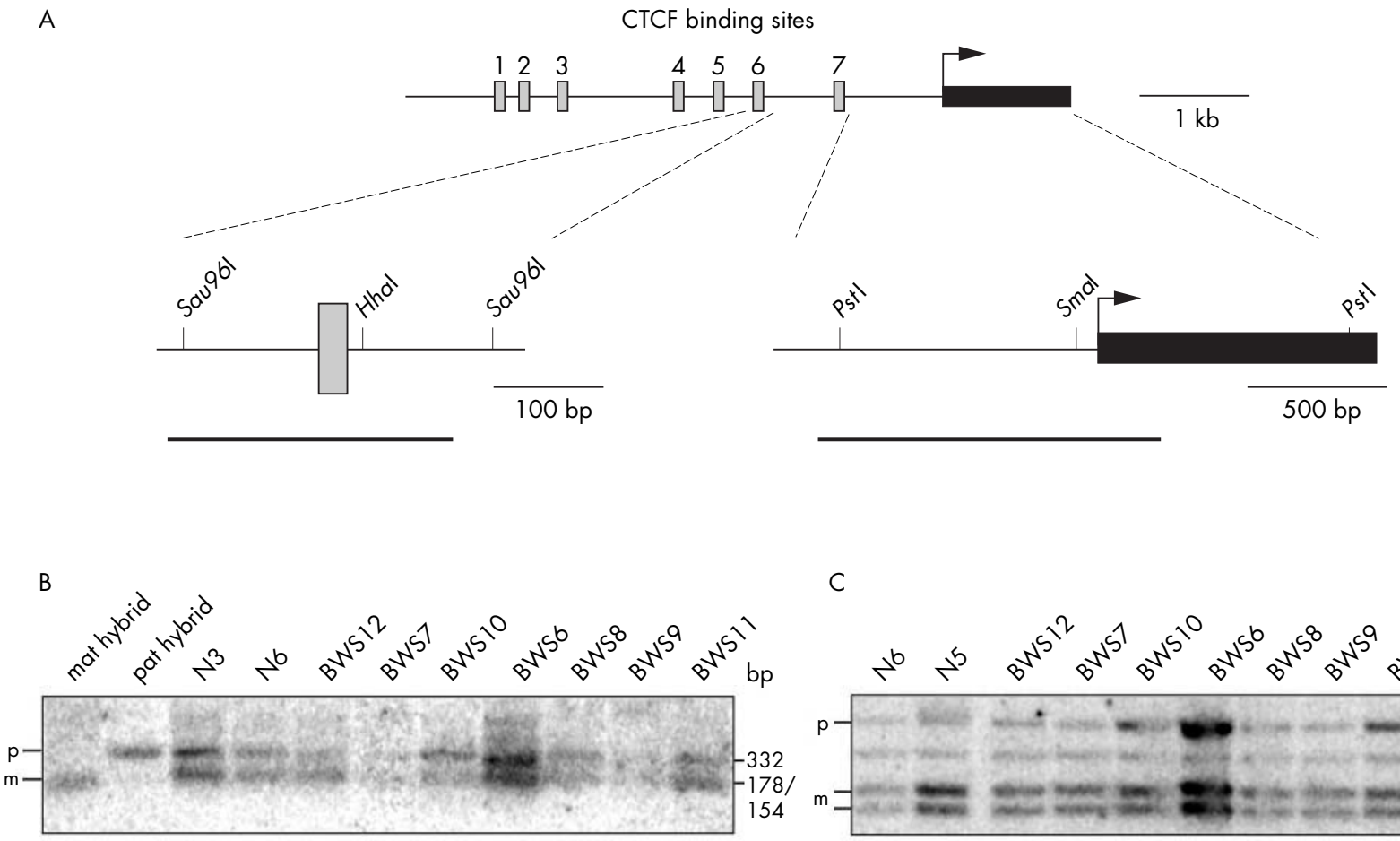

C

Figure 5 Methylation analysis of the region upstream of the $\mathrm{H} 19$ gene. (A) Map of the upstream region of the human $\mathrm{H} 19$ gene showing the locations of the seven CTCF binding sites and the positions of the two regions analysed for methylation; enlargements of these regions are shown below the map indicating the position of the methylation sensitive restriction enzymes (Hhal and Smal) used in the analysis. The heavy black lines below the enlargements represent the location of the hybridisation probes. (B) DNA from the indicated cell lines was digested with Sau96l and Hhal, electrophoresed and hybridised with a probe encompassing CTCF binding site No 6. The Hhal site was cleaved (unmethylated) in DNA from the somatic cell hybrid containing a maternally derived human chromosome 11 giving rise to a unresolvable doublet of 178/154 base pairs. The same site was resistant to cleavage (methylated) in DNA from a hybrid carrying a paternally derived chromosome 11 resulting in a 332 bp Sau96l fragment. All normal and patient samples showed both alleles suggestive of normal differential methylation at this site. (C) DNA from the indicated cell lines was digested with Pstl and Smal, electrophoresed, and hybridised with a probe encompassing the promoter region and part of exon 1 of the $\mathrm{H} 19$ gene. In each sample, the uncut $1.8 \mathrm{~kb}$ paternal allele as well as the cleavage products ( 1.0 and $0.8 \mathrm{~kb}$ ) of the maternal allele were visible, with no indication of hypermethylation. The band marked with an asterisk represents hybridisation of the probe to an adjacent Pstl fragment.

IGF2 is usually associated with hypermethylation upstream of the $H 19$ gene. ${ }^{37}$ Therefore we determined whether BWS patients with downregulated expression of CDKNIC also suffered an epimutation at the H19/IGF2 locus. Of the seven potential binding sites for the insulator protein CTCF in the human H19/IGF2 ICR (fig 5A), only site No 6 appears to be differentially methylated in normal tissue, and methylation at this site largely correlates with expression of IGF2 in somatic cell hybrids treated with 5 -aza-2'-deoxycytidine. ${ }^{38}$ Consistent with these results, we found that a single site for the methylation sensitive restriction enzyme HhaI next to CTCF binding site No 6 was unmethylated (cleaved) in a somatic cell hybrid carrying a maternally derived human chromosome 11, but methylated (uncut) in a hybrid containing a paternally derived chromosome 11 (fig 5B). Both digested and undigested alleles were observed in fibroblast DNA from normal individuals and BWS patients, including both subgroups with downregulation of $C D K N I C$, with no evidence of hypermethylation in any case (fig 5B). Also consistent with the lack of an epimutation in the H19 ICR in these patients was the finding of normal differential methylation at a SmaI site just upstream of the H19 start site (fig 5C), hypermethylation of which is associated with loss of imprinting at IGF2. ${ }^{37}$

\section{DISCUSSION}

Our results suggest the existence of at least three silencing mechanisms for CDKNIC. These alternative methods of inactivation of CDKNIC could all be functionally equivalent to loss of function mutations of CDKNIC. Regardless of the mechanism, downregulation of CDKNIC appears to be a common occurrence that may lead to $\mathrm{BWS}^{22}$ or, in some cases, contribute to cancer. ${ }^{72-3039-41}$ It should be noted, however, that reduced expression of CDKNIC in BWS patients does not generally appear to be associated with predisposition to cancer. Although one report suggests that LOM at KvDMRl, and presumably downregulation of CDKNIC, may be related to the incidence of non-Wilms' embryonyl tumours in BWS patients, ${ }^{42}$ several other studies suggest that biallelic expression of IGF2 associated with hypermethylation at the H19 ICR tracks with tumour development. ${ }^{21} 2425$

CDKNIC is silenced in association with promoter methylation in several tumour types..$^{27-30}$ This represents one mechanism for epigenetic silencing of CDKNIC. Although it is a possible mechanism for silencing CDKNIC in BWS, we did not observe hypermethylation of CDKNIC in any of the BWS patient samples available; however, a larger BWS patient sample size should be analysed to extend these results. Together, our results suggest the presence of distinct mechanisms in cancer and BWS for silencing of CDKNIC. It is evident that neither DNA methylation nor chromatin structure (at least as studied here) at the CDKNIC promoter region is altered in patients with LOM at KvDMRl and reduced expression of CDKNIC. Evidence complementing our results was recently provided by Soejima et al, ${ }^{41}$ who showed that downregulation of CDKNIC expression in some oesophageal cancers correlates with loss of $\mathrm{CpG}$ methylation and loss of histone $\mathrm{H} 3$ lysine 9 methylation at KvDMRl, but does 
not correlate with methylation or with histone modification at CDKNIC. Together, these results strongly support the view that LOM at KvDMRl is mechanistically related to the silencing of CDKNIC in these patients, and that these two epigenetic alterations are not simply independent manifestations of a domain-wide epimutation in 11p15.5.

The molecular mechanisms whereby KvDMRI regulates imprinting, in particular $C D K N I C$ imprinted expression, are not yet understood. It has been shown previously that loss of methylation at KvDMRl leads to biallelic expression of $K C N Q 1 O T 1$, an antisense (with respect to KCNQ1) non-coding RNA transcript (fig 1). ${ }^{19}$ It is possible that KCNQ1OT1 may function in a manner analogous to the Air transcript in the Igf $2 r$ locus, perhaps by facilitating the establishment and spreading of a domain-wide repressive chromatin structure. ${ }^{43}$ Indeed, Thakur et al have shown that, at least in an episomal context, the Kcnqlotl RNA may play a role in gene silencing. ${ }^{44}$ Enhancer blocking assays suggest that KvDMRl may function as a methylation sensitive insulator or as a bidirectional silencer, or both. ${ }^{15-18}$ Our finding that the chromatin structure of the CDKNIC promoter does not appear to be altered in BWS patients with LOM at KvDMRI and downregulation of CDKNIC argues against the establishment of domain-wide repressive chromatin on the maternal chromosome caused by the epimutation at KvDMRl. One could therefore speculate that, at least in some instances, KvDMRl may function like the H19 ICR (that is, as a chromatin insulator).

On the other hand, CDKNIC can be silenced by a KvDMRI independent mechanism. We identified three patients with normal methylation at KvDMRl but with low levels of CDKNIC expression. In the two patients analysed, we observed repressive chromatin modifications at the CDKNIC promoter region. Our analysis included antibodies for only three chromatin modifications, namely dimethyl H3-K4, dimethyl H3-K9, and HPl $\gamma$; nevertheless, enrichment of dimethyl H3-K9 and HPl $\gamma$, and depletion of dimethyl H3-K4 were clearly seen in this class of BWS patients. We therefore propose that the altered chromatin structure at the CDKNIC promoter region is associated with the downregulation of CDKNIC levels in these two patients. Whether reduced expression is caused by the acquisition of repressive chromatin at the promoter, or if the altered chromatin is a result of silencing by another mechanism, remains to be determined. Although studies both in cell culture and in the mouse indicate that reduction in the expression level of CDKNIC can be associated with excess IGF2 levels, ${ }^{36} 45$ real time RT-PCR analyses do not show increased levels of IGF2 expression in these patients, nor in BWS patients with LOM at KvDMRl (data not shown). Furthermore, Southern analysis at two loci known to be associated with LOI at IGF2 demonstrated normal methylation in both groups of BWS patients with reduced CDKNIC levels. These results argue that the repression of CDKNIC expression in BWS patients with normal methylation at KvDMRl is not related to overexpression of IGF2. Moreover, these finding suggest that it is not necessary to disrupt the expression of both CDKNIC and IGF2 in order to manifest BWS.

The aetiology of BWS is complex, with several different genetic and epigenetic mechanisms. ${ }^{1}$ However, the molecular defect in $10-20 \%$ of patients has yet to be established. Although the sample size is small, our finding of reduced levels of CDKNIC expression in three of six non-UPD BWS patients with normal methylation at KvDMRl suggests that a substantial proportion of these cases may show an aberrant chromatin structure at the CDKNIC promoter. Further studies are necessary to fully understand the mechanisms responsible for the change in histone modification in the CDKNIC promoter region and the impact of these modifications on chromatin structure and transcriptional regulation.

\section{ACKNOWLEDGEMENTS}

We thank Gail Graham and Vazken M Der Kaloustian for BWS patient cell lines. We also thank Dr Lionel Coignet for the Raji DNA. In addition, we thank Michelle Detwiler for help with algorithms in the DNA sequencing software as well as Rahul Patel and Ganesh Shankar for help with macros. Finally, we thank Dr Andrew Hoffman for kindly sharing chromatin antibodies. This research used core facilities supported in part by RPCI's NCI funded Cancer Center Support Grant, CAl6056. This work was supported by a grant from the Association for Research of Childhood Cancer (AROCC) and NCI/ $\mathrm{NIH}$ grant CA89426 to MJH.

\section{Authors' affiliations}

N Diaz-Meyer, Y Yang, M J Higgins, Department of Cancer Genetics, Roswell Park Cancer Institute, Buffalo, New York, USA

S N Sait, Cytogenetics Laboratory, Roswell Park Cancer Institute E R Maher, Section of Medical and Molecular Genetics, Department of Paediatrics and Child Health, University of Birmingham, Birmingham, UK

Competing interests: none declared

\section{REFERENCES}

1 Maher ER, Reik W. Beckwith-Wiedemann syndrome: imprinting in clusters revisited. J Clin Invest 2000;105:247-52.

2 Lee MH, Reynisdottir I, Massague J. Cloning of p57KIP2, a cyclin-dependent kinase inhibitor with unique domain structure and tissue distribution. Genes Dev 1995;9:639-49.

3 Matsuoka S, Edwards MC, Bai C, et al. p57KIP2, a structurally distinct member of the p21CIP1 Cdk inhibitor family, is a candidate tumor suppressor gene. Genes Dev 1995;9:650-62.

4 Zhang $\mathbf{P}$, Liegeois $\mathrm{N}$, Wong $\mathrm{C}$, et al. Altered cell differentiation and proliferation in mice lacking p57KIP2 indicates a role for in BeckwithWiedemann syndrome. Nature 1997;387:151-8.

5 Yan Y, Frisen J, Lee M-H, et al. Ablation of the CDK inhibitor p57KIP2 results in increased apoptosis and delayed differentiation during mouse development. Genes Dev 1997:11:973-83.

6 Chung WY, Yuan L, Feng L, et al. Chromosome 11 p15.5 regional imprinting: comparative analysis of KIP2 and H19 in human tissues and Wilms' tumors. Hum Mol Genet 1996;5:1101-8.

7 Hatada I, Inazawa J, Abe T, et al. Genomic imprinting of human p57KIP2 and its reduced expression in Wilms' tumors. Hum Mol Genet 1996:5:783-8.

8 Hatada I, Ohashi H, Fukushima Y, et al. An imprinted gene p57KIP2 is mutated in Beckwith-Wiedemann syndrome. Nat Genet 1996;14:171-3.

9 O'Keefe D, Dao D, Zhao L, et al. Coding mutations in p57KIP2 are present in some cases of Beckwith-Wiedemann syndrome but are rare or absent in Wilms tumors. Am J Hum Genet 1997;61:295-303.

10 Lam WW, Hatada I, Ohishi S, et al. Analysis of germline CDKN1C (p57KIP2) mutations in familial and sporadic Beckwith-Wiedemann syndrome (BWS) provides a novel genotype-phenotype correlation. J Med Genet 1999;36:518-23.

11 Arney KL. H19 and lgf2 - enhancing the confusion? Trends Genet 2003; 19:17-23.

12 Horike S, Mitsuya K, Meguro M, et al. Targeted disruption of the human LIT1 locus defines a putative imprinting control element playing an essential role in Beckwith-Wiedemann syndrome. Hum Mol Genet 2000;9:2075-83.

13 Fitzpatrick GV, Soloway PD, Higgins MJ. Regional loss of imprinting and growth deficiency in mice with a targeted deletion of KvDMR1. Nat Genet 2002;32:426-31.

14 Lewis A, Mitsuya K, Umlauf D, et al. Imprinting on distal chromosome 7 in the placenta involves repressive histone methylation independent of DNA methylation. Nat Genet 2004;36:1291-5.

15 Kanduri C, Fitzpatrick G, Mukhopadhyay R, et al. A differentially methylated imprinting control region within the Kcnql locus harbors a methylationsensitive chromatin insulator. J Biol Chem 2002;277:18106-10.

16 Mancini-DiNardo D, Steele SJ, Ingram RS, et al. A differentially methylated region within the gene Kcnql functions as an imprinted promoter and silencer. Hum Mol Genet 2003; 12:283-94.

17 Thakur N, Kanduri M, Holmgren C, et al. Bidirectional silencing and DNA methylation-sensitive methylation-spreading properties of the $\mathrm{Kcnq} \mathrm{l}$ imprinting control region map to the same regions. J Biol Chem 2003;278:9514-19.

18 Du M, Beatty LG, Zhou W, et al. Insulator and silencer sequences in the imprinted region of human chromosome 11 p15.5. Hum Mol Genet 2003; 12:1927-39.

19 Lee MP, DeBaun MR, Mitsuya K, et al. Loss of imprinting of a paternally expressed transcript, with antisense orientation to KVLQT1, occurs frequently in Beckwith-Wiedemann syndrome and is independent of insulin-like growth factor II imprinting. Proc Natl Acad Sci USA 1999;96:5203-8.

20 Smilinich NJ, Day CD, Fitzpatrick GV, et al. A maternally methylated CpG island in KVLQT1 is associated with an antisense paternal transcript and loss of imprinting in Beckwith-Wiedemann syndrome. Proc Natl Acad Sci USA 1999;96:8064-9.

21 Engel JR, Smallwood A, Harper A, et al. Epigenotype-phenotype correlations in Beckwith-Wiedemann syndrome. J Med Genet 2000;37:921-6. 
22 Diaz-Meyer N, Day CD, Khatod K, et al. Silencing of CDKN1C (p57(KIP2)) is associated with hypomethylation at KvDMR1 in Beckwith-Wiedemann syndrome. J Med Genet 2003;40:797-801.

23 Tokino T, Urano T, Furuhata T, et al. Characterization of the human p57KIP2 gene: alternative splicing, insertion/deletion polymorphisms in VNTR sequences in the coding region, and mutational analysis. Hum Genet 1996;97:625-31.

24 Bliek J, Maas SM, Ruijter JM, et al. Increased tumour risk for BWS patients correlates with aberrant $\mathrm{H} 19$ and not KCNQ1OT1 methylation: occurrence of KCNQ1OT1 hypomethylation in familial cases of BWS. Hum Mol Genet 2001; 10:467-76.

25 DeBaun MR, Niemitz EL, McNeil DE, et al. Epigenetic alterations of $\mathrm{H} 19$ and LIT1 distinguish patients with Beckwith-Wiedemann syndrome with cancer and birth defects. Am J Hum Genet 2002;70:604-11.

26 Clark SJ, Harrison J, Paul CL, et al. High sensitivity mapping of methylated cytosines. Nucleic Acids Res 1994;22:2990-7.

27 Kikuchi T, Toyota M, Itoh F, et al. Inactivation of p57KIP2 by regional promoter hypermethylation and histone deacetylation in human tumors. Oncogene 2002;21:2741-9.

28 Li Y, Nagai H, Ohno T, et al. Aberrant DNA methylation of p57(KIP2) gene in the promoter region in lymphoid malignancies of B-cell phenotype. Blood 2002; 100:2572-7.

29 Shin JY, Kim HS, Park J, et al. Mechanism for inactivation of the KIP family cyclin-dependent kinase inhibitor genes in gastric cancer cells. Cancer Res 2000:60:262-5.

30 Shen L, Toyota M, Kondo Y, et al. Aberrant DNA methylation of p57KIP2 identifies a cell-cycle regulatory pathway with prognostic impact in adult acute lymphocytic leukemia. Blood 2003;101:4131-6.

31 Xiong Z, Laird PW. COBRA: a sensitive and quantitative DNA methylation assay. Nucleic Acids Res 1997;25:2532-4.

32 Gabriel JM, Higgins MJ, Gebuhr TC, et al. A model system to study genomic imprinting of human genes. Proc Natl Acad Sci USA 1998;95:14857-62.

33 Strahl BD, Ohba R, Cook RG, et al. Methylation of histone H3 at lysine 4 is highly conserved and correlates with transcriptionally active nuclei in Tetrahymena. Proc Natl Acad Sci USA 1999;96:14967-72.
34 Minc E, Courvalin JC, Buendia B. HPlgamma associates with euchromatin and heterochromatin in mammalian nuclei and chromosomes. Cytogenet Cell Genet 2000;90:279-84.

35 Lachner M, O'Carroll D, Rea S, et al. Methylation of histone H3 lysine 9 creates a binding site for HP1 proteins. Nature 2001;410:116-20.

36 Caspary T, Cleary MA, Perlman EJ, et al. Oppositely imprinted genes p57(Kip2) and lgf2 interact in a mouse model for Beckwith-Wiedemann syndrome. Genes Dev 1999;13:3115-24.

37 Reik W, Brown KW, Schneid H, et al. Imprinting mutations in the BeckwithWiedemann syndrome suggested by altered imprinting pattern in the IGF2H19 domain. Hum Mol Genet 1995;4:2379-85.

38 Takai D, Gonzales FA, Tsai YC, et al. Large scale mapping of methylcytosines in CTCF-binding sites in the human $\mathrm{H} 19$ promoter and aberrant hypomethylation in human bladder cancer. Hum Mol Genet 2001; 10:2619-26.

39 Thomson J, Reese K, DeBaun M, et al. Reduced expression of the cyclindependent kinase gene p57-KIP2 in Wilms' tumor. Cancer Research 1996:56:5723-27.

40 Schwienbacher C, Gramantieri L, Scelfo R, et al. Gain of imprinting at chromosome 11p15: A pathogenetic mechanism identified in human hepatocarcinomas. Proc Natl Acad Sci USA 2000;97:5445-9.

41 Soejima H, Nakagawachi T, Zhao W, et al. Silencing of imprinted CDKN1C gene expression is associated with loss of $\mathrm{CpG}$ and histone $\mathrm{H} 3$ lysine 9 methylation at DMR-LIT1 in esophageal cancer. Oncogene 2004;23:4380-8.

42 Weksberg R, Nishikawa J, Caluseriu O, et al. Tumor development in the Beckwith-Wiedemann syndrome is associated with a variety of constitutional molecular 11 p15 alterations including imprinting defects of KCNQ1OT1. Hum Mol Genet 2001;10:2989-3000.

43 Sleutels F, Zwart R, Barlow DP. The non-coding Air RNA is required for silencing autosomal imprinted genes. Nature 2002;415:810-13.

44 Thakur N, Tiwari VK, Thomassin H, et al. An antisense RNA regulates the bidirectional silencing property of the kcnql imprinting control region. Mol Cell Biol 2004:24:7855-62.

45 Grandjean V, Smith J, Schofield PN, et al. Increased IGF-II protein affects p57kip2 expression in vivo and in vitro: implications for BeckwithWiedemann syndrome. Proc Natl Acad Sci USA 2000;97:5279-84.

\section{Call for papers}

11 th European Forum on Quality Improvement in Health Care

26-28 April 2006, Prague, Czech Republic

Deadline 30 September 2005.

For further information and to submit online go to: www.quality.bmipg.com 